\title{
La enseñanza práctica en Fonoaudiología a través de modelos tridimensionales: una alternativa prometedora
}

\section{Theoretical-Practical Teaching in Speech-Language Pathology through three-dimensional models: a promising alternative}

David Esquível-Oliveira

Instituto de Ciências Biomédicas,

Centro de Ciências da Saúde, Universidade Federal do Rio de Janeiro

\section{Celia Palmero}

Instituto de Ciências Biomédicas,

Centro de Ciências da Saúde, Universidade Federal do Rio de Janeiro

Bruna Gonçalves

Instituto de Ciências Biomédicas,

Centro de Ciências da Saúde,

Universidade Federal do Rio de Janeiro

\section{Elenice Correa-Gillieron} Instituto de Ciências Biomédicas,

Centro de Ciências da Saúde, Universidade Federal do Rio de Janeiro
Contacto con el autor: Elenice M. Correa-Gillieron

Rio de Janeiro - Brasil Correo-e: corgillieron@ufrj.br

Recibido: $17 / 10 / 2017$ Aceptado: 04/06/2018

\section{RESUMEN}

El análisis de cuestionarios respondidos por alumnos de la disciplina Sistemas Sensoriales del curso de Fonoaudiología mostró que había dificultades para entender la histología de las regiones anatómicas del oído. Se estableció que la compresión de la histología demandaba una actividad didáctica complementaria. Por ello, se decidió crear una clase teórico-práctica diferenciada (CTP) usando un modelo tridimensional (3D) del oído humano y preparaciones histológicas de sus regiones. El modelo es una réplica anatómica muy aumentada del oído humano que permite la manipulación exploratoria de modo más eficaz que con piezas anatómicas clásicas. La CTP fue implementada en los dos semestres de 2014. La evaluación de la CTP incluyó un cuestionario de opiniones, más un cuaderno de actividades. Los resultados mostraron medias finales de mejores desempeños en ese año respecto al 2013, donde no fue dada este tipo de clase teórico-práctica. Se concluye que la CTP, asociada al modelo tridimensional y las explicaciones teóricas-prácticas de las preparaciones histológicas, permitió una mejor comprensión del contenido enseñado e introdujo una metodología más eficiente para el proceso de enseñanza-aprendizaje de un tema tan complejo así como importante para la formación académica de los estudiantes de Fonoaudiología. Adicionalmente, a partir de esta experiencia teórico-práctica se ha demostrado, una vez más, la importancia de la relación personal y profesional entre el profesor, preparador estudiantil y los alumnos para la calidad del trabajo académico.

Palabras clave: Fonoaudiología, histología, modelado 3D, clase práctica.

\section{ABSTRACT}

We designed a differentiated theoretical-practical class (CTP) after analyzing questionnaires (Qs) answered by Speech-therapy students that attended the course "Sensory Systems". The Qs showed difficulties related with understanding the practical histology of the sensory systems, as well as problems to correlate it with the learning of the anatomy. We therefore established the to implement an auxiliary didactic activity (CTP). We used a three-dimensional model (3D) AS-001, of the University Extension Project (3D Museum). AS-001, is an enlarged anatomical replica of the human ear which is much more efficient for exploratory manipulation much than anatomical modeling. The assessment of CTP included a questionnaire and a notebook of activities. The CTP was implemented in both semesters during the year 2014. Results showed improvements when compared to 2013's data, when this type of theoretical-practical contents were not implemented. We conclude that the association of the three - dimensional model along with histological analysis of the parts of the ear effectively improved students' understanding of the subject. We therefore consider that CTP is a suitable methodology for the teaching-learning process of a complex, important subject for the academic training of Speech-therapy undergraduate students. Our experience allowed us to determine how important the personal and professional relationship between teachers and students is to improve academic work.

Keywords: Speech-therapy, histology, 3D model, practical courses. 


\section{Introducción}

Actualmente, las discusiones sobre las formas de enseñar se han producido en todos los niveles educativos. Este cuestionamiento sobre qué y cómo enseñar mejor ha generado una serie de estudios que buscan despertar en los educadores el deseo de repensar y reevaluar paradigmas pedagógicos que parecen insuficientes para responder a los nuevos e inquietantes desafíos del mundo moderno (Augusto et al., 2016). Formar mentes calificadas para enfrentar los retos de un mundo globalizado es la principal preocupación de las instituciones educativas en los diferentes niveles y modalidades: preescolar, básica, media y superior. En la actual visión educacional, el propio concepto de sala de clases se ha modificado y ampliado, desde la tradicional concepción de "espacio destinado a la enseñanza", a la de "cualquier espacio donde se (re)construye el proceso de enseñanza-aprendizaje". Esta última concepción implica una visión educacional más dinámica. El nuevo panorama conlleva la necesidad de introducir modificaciones en el clásico eje pedagógico donde un profesor "soberano" conducía el proceso de enseñanza para un receptáculo de conocimientos (el o la estudiante) (Cunha, 2013) que supuestamente nada sabía. Los métodos auxiliares en este tipo de transmisión de conocimiento eran, invariablemente, el libro texto, formulación de cuestionarios y trabajos creados por el profesor.

En la Universidad Federal do Rio de Janeiro (UFRJ) el curso de Fonoaudiología de la Facultad de Medicina incluye en su estructura curricular clases prácticas, específicamente en lo que se refiere a temas sobre Fonoaudiología. Sin embargo, algunas disciplinas básicas y generales (fundamentales para la preparación del alumno para las disciplinas de formación profesional) carecen de actividades prácticas en Histología, como es el caso de la disciplina Programa Curricular Interdepartamental (PCI) de los Sistemas Sensoriales. Esta disciplina se imparte en el 20 período/diurno, y envuelve la Histología (base teórica), Embriología (base teórica), Anatomía (base teórica y práctica) y Fisiología (base teórica) de los oídos externos, medio e interno. En consecuencia, es un tema complejo de abordar en las clases.

Aprender sobre el oído es fundamental para la Audiología, rama importante de la Fonoaudiología que lleva al fonoaudiólogo a prepararse para poder así ayudar a pacientes con deficiencias auditivas, en las situaciones cotidianas. Es muy importante la actuación fonoaudiológica en el área de la rehabilitación, que involucra también la rehabilitación vestibular. Los fonoaudiólogos actúan en el área de rehabilitación vestibular desde finales de la década de 70 (Mor \& Fragoso, 2012). Su participación complementa y enriquece el tratamiento del médico, cuya consecuencia redunda en un mejor tratamiento para el paciente. Por lo tanto, conocer sobre la estructura (macro y microscópicamente) del oído es, desde una perspectiva profesional, relevante para el fonoaudiólogo.

La ausencia de una práctica histológica en el PCl nos motivó en el año 2013 a realizar una encuesta de satisfacción con los alumnos que ya habían cursado este $\mathrm{PCl}$, a fin de determinar las posibles deficiencias en el proceso de enseñanza-aprendizaje del tema "el 
oído". Verificamos que los alumnos no logran una clara visión espacial y tridimensional de la estructura del oído. También, observamos que no consiguieron correlacionar de forma clara las diferentes partes del oído con su organización histológica. Ello a pesar de que existe un una unidad destinada a abordar desde la teoría y la práctica la anatomía del oído. Para esto se utiliza un atlas anatómico sobre sistema auditivo y vestibular (Correa, 2011), que contiene información, imágenes y esquemas. Además, se realizan otras actividades didácticas con videos y diapositivas que muestran la estructura del oído.

Después de una cuidadosa evaluación de las opiniones de los estudiantes determinamos la necesidad de introducir una actividad práctica que permitiese una mejor comprensión de la estructura del oído. Inferimos que la dificultad se originaba en el hecho de no considerar clases prácticas de la unidad de Histología. Los estudiantes manifestaron también que el abordaje anatómico no estaría ofreciendo la base adecuada para la comprensión de los diferentes componentes del oído. Lo anterior debido al número deficiente de piezas anatómicas o al inadecuado estado de conservación.

A partir de los antecedentes mencionados, el año 2014 se creó una clase teórico-práctica (CTP) que buscaba ampliar la participación activa de los alumnos en el propio proceso de enseñanzaaprendizaje. Para tal fin, escogimos usar un modelo tridimensional que exhibe las regiones anatómicas del oído externo, medio e interno, en lugar de una exposición de piezas anatómicas clásicas.

El uso de modelos 3D en la universidad es una práctica antigua, que por años sirvió para la enseñanza de órganos y sistemas. No sólo se utilizó en la UFRJ (durante las décadas del 60 y 70) sino también en otras universidades del país y del mundo entero (Augusto et al., 2016). En lo que respecta a la UFRJ, esta práctica se fue abandonando debido a la mala utilización de los modelos y a las condiciones precarias de durabilidad, conservación y preservación de los modelos del acervo. Además, existía gran dificultad de encontrar técnicos capaces de esculpir reproducciones excepcionalmente realistas de las estructuras anatómicas.

Para cumplir el objetivo de la CTP al modelo previamente mencionado se asociaron preparaciones histológicas específicas (preparaciones histológicas permanentes) que correspondían a los tejidos constituyentes de cada una de las regiones anatómicas del modelo. Con esta innovación metodológica esperábamos que, de una forma práctica, los estudiantes consolidaran sus conocimientos teóricos sobre los tres oídos, desarrollados previamente en la sala de clase. Adoptamos una estrategia didáctica para enseñar la práctica de Anatomía e Histología de manera correlacionada, propiciando así un proceso de enseñanza-aprendizaje por asociación.

El presente trabajo tiene como objetivo presentar la experiencia de enseñanza que se realizó con la estrategia metodológica expuesta anteriormente. La estrategia buscaba potenciar el proceso de enseñanza-aprendizaje a través de la estimulación multisensorial del alumno durante las clases prácticas de histología (en especial la histología del oído) de la Carrera de Fonoaudiología. 


\section{Descripción de la experiencia}

El modelo tridimensional AS-001 integra el acervo del proyecto de Extensión Universitaria llamado Museo $3 D$ de la UFRJ. Este modelo es una réplica exacta del oído humano y sus divisiones (Fig. 1). Sin embargo, sus dimensiones son superiores a las reales (ver tabla 1). El AS-001, pintado con colores diferentes para cada región (Fig. 1), fue tallado lo más próximo posible a las características originales de este órgano, reproduciendo así los lugares donde existe piel y hueso.

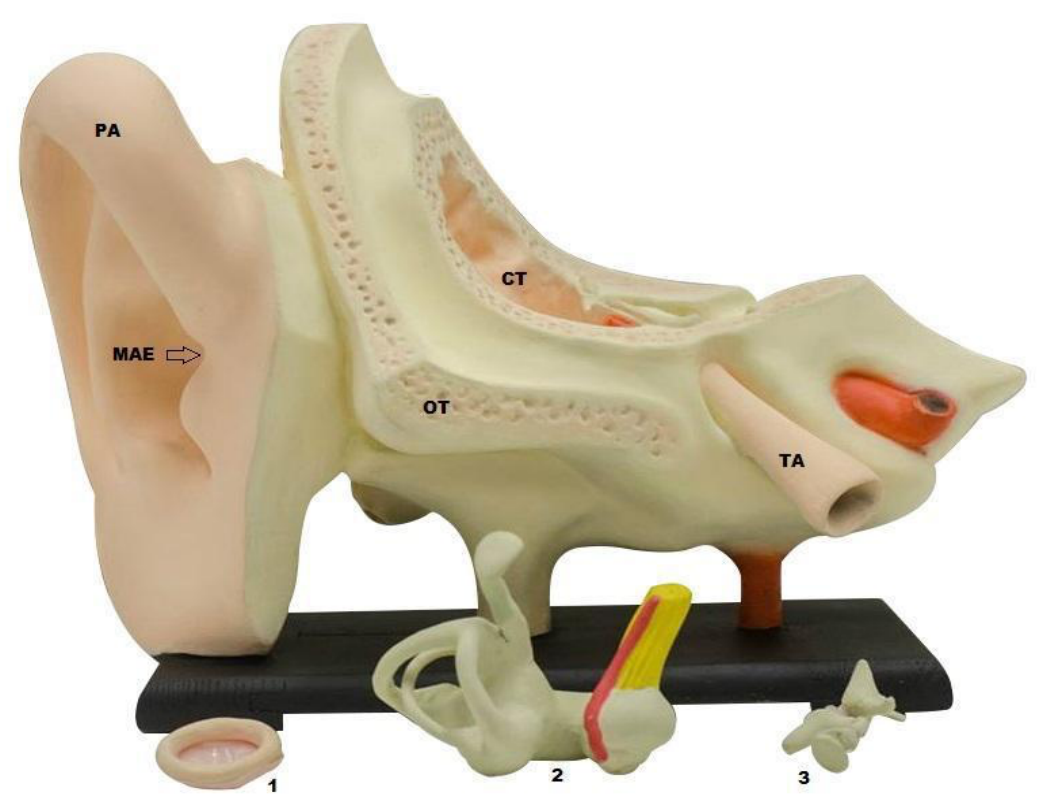

Figura 1. El modelo tridimensional AS-001, representa el oído humano: PA: pabellón auricular; MAE: meato acústico externo; CT: cavidad timpánica; OT: hueso temporal; TA: tubo faringotimpánico; 1: membrana timpánica (tímpano); 2; oído interno; 3 : cadena de huesecillos.

Tabla 1

Algunas medidas del modelo tridimensional del oído humano.

\begin{tabular}{lcc}
\hline Área & Medida real & Medida en el modelo \\
\hline Pabellón auricular- Eje hélice-mandibular & $5 \mathrm{~cm}$ & $17 \mathrm{~cm}$ \\
Cavidad timpánica (anchura) & $2 \mathrm{~cm}$ & $6,8 \mathrm{~cm}$ \\
Del tímpano a la ventana oval- En el oído medio en tamaño real & $6-8 \mathrm{~mm}$ & $2,8 \mathrm{~cm}$ \\
Huesecillos - estribo & $0,25 \mathrm{~cm}$ & $1,5 \mathrm{~cm}$ \\
Membrana timpánica (diámetro) & $1 \mathrm{~cm}$ & $4 \mathrm{~cm}$ \\
Diámetro del meato acústico externo & $7 \mathrm{~mm}$ & $3,2 \mathrm{~cm}$
\end{tabular}


Como La CTP fue aplicada por un preparador estudiantil y por el profesor en los dos semestres del año 2014 (primer semestre: 26 alumnos, y segundo semestre: 40 alumnos). Se buscaba reforzar las clases teóricas de Histología y Anatomía. Las actividades de la CTP se realizaron en el laboratorio de microscopia óptica y tuvo una duración aproximada de 2 horas y 45 minutos. La CTP fue desarrollada en tres etapas, con un intervalo de 10 minutos entre las etapas II y III. Además, aplicamos un cuestionario de satisfacción para evaluar el impacto de la actividad en el aprendizaje de las dos disciplinas abordadas (Anatomía e Histología). A continuación, se expone un resumen de las diferentes etapas.

\section{Etapa I. Presentación del modelo y aclaración de} posibles dudas (45 minutos)

En esta etapa el preparador estudiantil (Fig. 2A), explicó el modelo en 3D del oído humano (AS-001) con sus tres regiones: oído externo, oído medio y oído interno, alojadas en el hueso temporal (Fig. 1). El modelo facilitaba la visualización de los componentes del oído externo (pabellón auricular y meato auditivo externo), del oído medio (el tímpano, la cavidad timpánica con la cadena de huesecillos, y parte del tubo faringotimpánico), así como del oído interno (la cóclea, vestíbulo, canales semicirculares y parte del nervio auditivo). De este modo, el preparador estudiantil, asesorado por el profesor, recordó a los estudiantes la anatomía ya analizada con el uso del atlas anatómico. De igual manera, durante la explicación se realizaron preguntas a los alumnos que reforzaron la información sobre el tema ya enseñado en la clase teórica. Todos los estudiantes podían tomar anotaciones para el estudio posterior del tema discutido en esta etapa. Al manipular el modelo 3D de manera exploratoria, los estudiantes planteaban dudas que no habían surgido ni en la clase teórica ni en la práctica anatómica tradicional. Esta última siempre era limitada, ya sea por la escasez o por la baja calidad de conservación de las piezas anatómicas. El principal objetivo en esta etapa fue recordar contenidos previamente enseñados. Por lo tanto, fue un reforzamiento. 


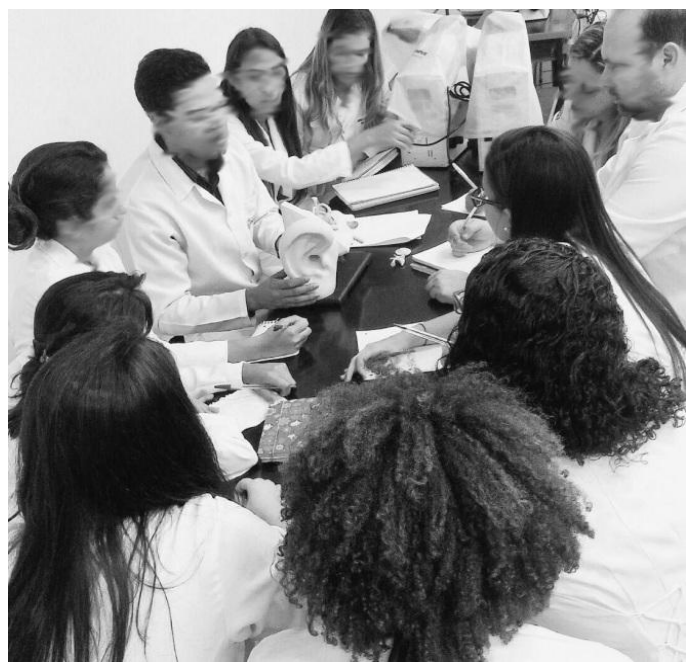

$2 \mathrm{~A}$

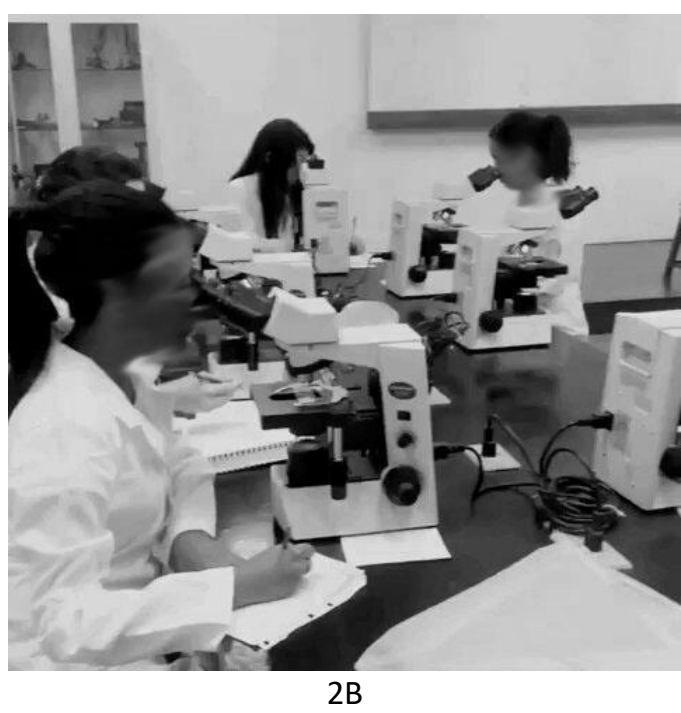

Figura 2. Alumnos en la clase teórico-práctica realizada en la sala de microscopia. A) Los alumnos atienden a la descripción del modelo tridimensional AS-001, realizada por el preparador estudiantil. B) Alumnos analizando al microscopio de luz las preparaciones histológicas correspondientes a los tejidos de las estructuras observadas en el modelo 3D (oído humano). Durante esta actividad, los estudiantes observan los tejidos después de la proyección y descripción de cada preparación histológica, realizadas por el profesor, a través del proyector localizado en la mesa principal.

\section{Etapa II. Observación de las preparaciones histológicas al microscopio de luz (60 minutos)}

Después del estudio práctico realizado con el modelo 3D, los estudiantes se dirigían a las mesas de trabajo para la observación y análisis de las preparaciones histológicas de cada región anatómica del modelo (Fig. 2B) a través de microscopios de luz binoculares (Zeiss Axio Lab). Durante esta etapa, el profesor describió toda la histología de las regiones observadas en el modelo AS-001, el que podía ser manipulado libremente por los alumnos con auxilio del preparador estudiantil. Durante la explicación histológica se proyectó cada preparación histológica a través de un microscopio principal acoplado a un ordenador, que a su vez se proyectaba en los monitores de cada mesa. Luego, los estudiantes observaron y analizaron las preparaciones histológicas a través de sus propios microscopios y tomaron notas personales sobre el tejido enseñado.
El principal objetivo en esta etapa fue el aprendizaje de la organización histológica de cada componente del oído y la correlación visual entre una región anatómica y su histología.

\section{Etapa III. Cuaderno de actividades (60 minutos)}

Después de las etapas I y II, hubo 10 minutos de intervalo que fueron empleados por los alumnos para volver a leer sus anotaciones antes de pasar a la etapa III. En esta etapa cada alumno recibió un Cuaderno de Actividades Prácticas (CAP) con preguntas e imágenes asociadas, referentes a lo que fue visualizado en la etapa II. Se les prohibió durante la etapa el uso de cualquier tipo de anotación realizada previamente. El principal objetivo de la presente etapa fue verificar el proceso de aprendizaje y el grado de comprensión obtenido en las etapas anteriores. 


\section{Evaluación de la experiencia}

Junto con cada CAP fue distribuido un Cuestionario de Satisfacción de la CTP (Fig. 3), con preguntas abiertas. Los alumnos tuvieron 20 minutos adicionales para responder dicho cuestionario. Los CAPs y los cuestionarios respondidos eran devueltos al preparador estudiantil al finalizar la clase.
Optamos por usar un cuestionario simplificado compuesto por siete preguntas cerradas. Las opciones de respuesta eran: sí, no, y no tengo conocimiento sobre este asunto (este último tuvo el mismo significado que, no tengo nada que declarar). Además, había una pregunta abierta.

Las preguntas del cuestionario son las siguientes:

1. ¿Tienes clases prácticas de anatomía en tu curso?

$\square$ si $\square$ no $\square$ no tengo conocimiento sobre ese asunto

2. ¿En caso de tener clases prácticas de anatomía en tu curso, las piezas anatómicas utilizadas son suficientes para la demanda de los alumnos?

$\square$ si $\square$ no $\square$ no tengo conocimiento sobre ese asunto

3. ¿Consideras que las piezas anatómicas en tu curso se encuentran en buen estado de conservación?

$\square$ si $\square$ no $\square$ no tengo conocimiento sobre ese asunto

4. ¿En el primer período del curso de Fonoaudiología son realizadas clases prácticas de histología, pero sólo sobre tejidos en general? ¿¿Fue pasado algún conocimiento sobre histología de oído?

$\square$ si $\quad \square$ no $\quad \square$ no tengo conocimiento sobre ese asunto

5. ¿Usted acepta que visualizar preparaciones histológicas asociadas al modelo tridimensional utilizado en la clase, mejoró la tuya aprendizaje acerca del tema?

$\square$ si $\square$ no $\square$ no tengo conocimiento sobre ese asunto

6. ¿Fue interesante el contacto directo y la manipulación de modelo 3D para tu proprio aprendizaje?

$\square$ si $\square$ no $\square$ no tengo conocimiento sobre ese asunto

7. ¿El contacto previo con el modelo 3D de oído ilustró el asunto e por esto mejoró tu percepción acerca del tema?

$\square$ si $\square$ no $\square$ no tengo conocimiento sobre ese asunto

Pregunta: Después de participar de la clase teórico-práctica, qué piensas acerca de una clase práctica con la ayuda de un modelo tridimensional y sobre la visualización al microscopio de luz de preparaciones histológicas relacionadas.

Sea breve en su respuesta.

Figura 3. Modelo del Cuestionario aplicado a los alumnos del Curso de Fonoaudiología de la UFRJ, en los dos semestres de 2014, a fin de evaluar la opinión de los alumnos con respecto a la metodología utilizada en la clase teórico-práctica del oído humano. 


\section{Resultados y Discusión}

En el año 2013 se estableció, mediante la encuesta realizada a los alumnos del PCI de Sistemas Sensoriales, que ellos presentaban dificultades para relacionar las regiones anatómicas del oído con su organización histológica. La razón principal era un desconocimiento práctico de la histología del órgano. Lo anterior afectaba el aprendizaje total y efectivo de la morfología de los sistemas auditivo y vestibular, dificultando a su vez la comprensión de su fisiología. Estos hallazgos están en concordancia con lo planteado por Candau (2011) quien afirma que los estudiantes del área de la salud presentan gran dificultad para comprender las estructuras tridimensionales explicadas en clases. Fue así que, basados en estas opiniones y considerando la importancia del conocimiento de estos sistemas para la Fonoaudiología, resolvimos aplicar una metodología diferenciada como acción educacional, desarrollando la CTP.

El punto básico y favorable para la inclusión de esta actividad práctica fue tener presente de que el entendimiento sobre el oído, implica conocer los diferentes aspectos morfológicos. Ello incluye la disposición anatómica y la cito-arquitectura de este órgano, lo que permite una comprensión plena de su función. Sin embargo, la ausencia de una práctica histológica en este $\mathrm{PCl}$ siempre dificultó la correlación entre la cito-arquitectura y la anatomía de las diferentes partes del oído. Como consecuencia, el pre-conocimiento que era necesario para la comprensión de la fisiología de los sistemas auditivo y vestibular estaba significativamente afectado, lo que limitaba la comprensión de la morfología del oído.

En la CTP se asociaron dos metodologías, el uso del modelo 3D para enseñar anatomía y la observación de preparaciones histológicas permanentes para comprender mejor cada uno de los constituyentes y cada región anatómica que estaba representada en el modelo.

El análisis de los cuestionarios de evaluación de los estudiantes que tuvieron la CTP mostró la aprobación de las metodologías didácticas utilizadas, la aceptación del modelo tridimensional y de la práctica de histología como una forma de cubrir la brecha en el aprendizaje del tema (Fig. 4). Las respuestas de los cuestionarios fueron muy importantes para evaluar la eficacia de la CTP, evidenciando la relevancia que el modelo tridimiensional tuvo para los estudiantes. 
CUESTIONARIO 10 SEMESTRE 2014

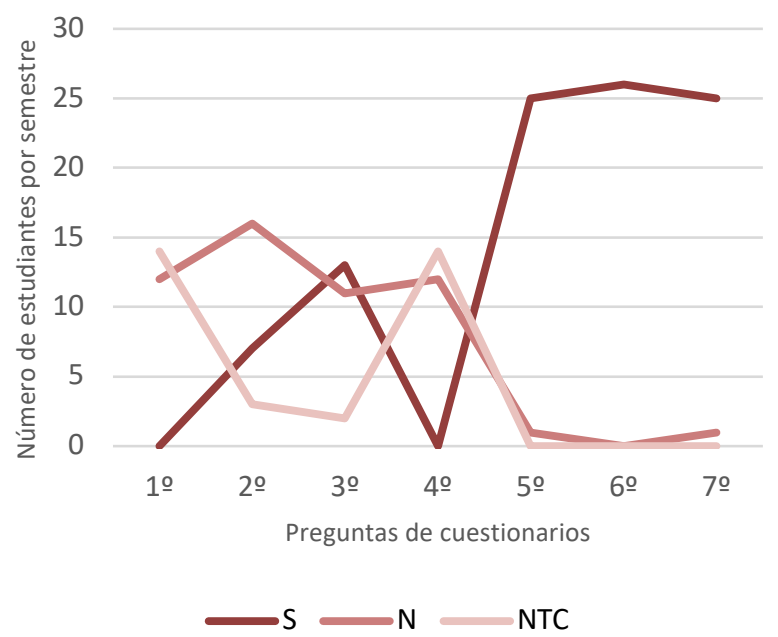

CUESTIONARIO 2 SEMESTRE 2014

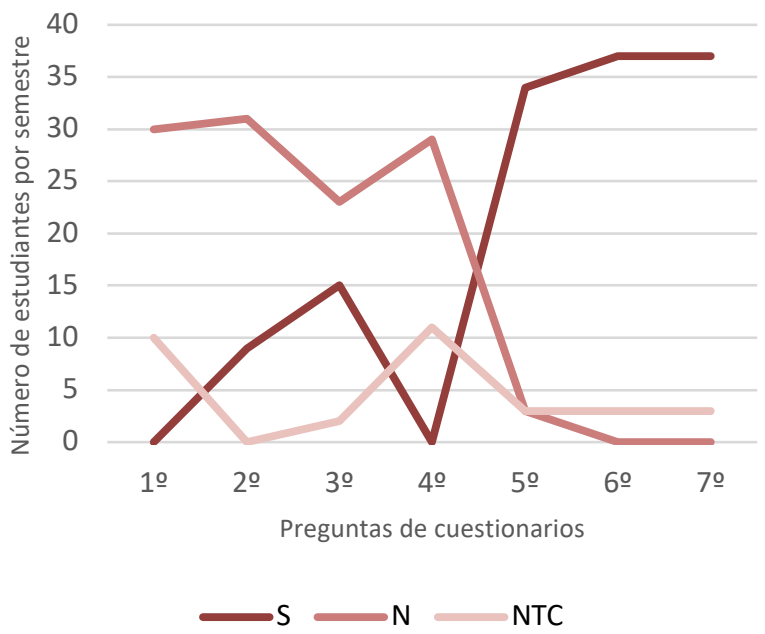

Figura 4. Gráficos representativos de las respuestas de los estudiantes a los cuestionarios usados en el primer y segundo semestre de 2014. $S=$ Si; $N=$ No; NTC = no tengo conocimiento. Cantidad de matrículas: 26 alumnos en primer semestre y 40 alumnos en segundo semestre).

Los comentarios a la pregunta libre indican que por ser una réplica exacta de una pieza anatómica pero con dimensiones mayores, el modelo AS-001 se convierte en un recurso muy apropiado y didáctico para la CTP.

La libertad para manipular a su antojo el modelo anatómico fue muy bien recibida por los estudiantes. Además el modelo permite un estudio más interactivo. Esto significó una experiencia mucho más agradable que estudiar con una pieza anatómica clásica. En efecto, esta representación tridimensional del oído permite la manipulación del modelo de una manera simple y adecuada para un estudio exploratorio. Además, no presenta los inconvenientes de la manipulación libre de una pieza anatómica post-mortem, la que exhibe artefactos causados por las sustancias fijadoras y necesita ser manipulada con bastante precaución, sin contar los aspectos emocionales implicados con el uso de piezas provenientes de humanos muertos. Cabe señalar que la inclusión que hicimos del modelo en $3 \mathrm{D}$, confirma al igual que los autores Oliveira et al. (2012), que las clases prácticas son situaciones de enseñanza-aprendizaje muy valiosas para los estudiantes pues traen innovaciones a estas clases.

Otro aspecto importante fue que la visualización de las partes anatómicas aumentadas en el modelo, favoreció el aprendizaje de los alumnos. Ello propició una discusión más interactiva y mantuvo la concentración durante la explicación, sin las distracciones comunes de las clases prácticas producidas por el uso de material cadavérico. Esto facilitó el proceso de enseñanza-aprendizaje y por ello fue más rápido y eficiente. Como el modelo se mantuvo en el aula, los estudiantes, además de tomar notas durante la explicación del profesor, manipulaban el modelo varias veces para realizar nuevos análisis y aclarar sus dudas.

Comprender cualquier objeto implica observarlo en sus relaciones con otros objetos $y$ en sus 
diferentes planos, de modo que los significados formen ejes de relaciones que se articulan en redes (Alarcão, 2015). De acuerdo con Luria (1981) la asociación de elementos que se relacionan entre sí es lo que conduce a un aprendizaje efectivo. Toda la acción educativa desarrollada se sustenta en los planteamientos previos. Lo anterior dado que las actividades se realizaron en una sala apropiada para la práctica histológica (el laboratorio de histología) en la que modelo $3 D$ se encontraba en el mismo lugar que las preparaciones histológicas representativas de los tejidos y estructuras descritos en las diferentes partes del modelo. Ello posibilitó que los alumnos asociaran de manera rápida la histología que estaba siendo aprendida y la anatomía revisada en el modelo AS-001. Además, facilitó que los estudiantes discutieran más y que sus dudas se clarificaran. Todo lo anterior permitió que obtuvieran conclusiones finales de una forma más práctica, interactiva e inteligible, sin que fueran necesarias la memorización y la visualización tridimensional comprensible, actividades propias de una clase tradicional (de Lima et al., 2015). Sin duda, el modelo 3D facilitó la comprensión y fue considerado una metodología de trabajo innovadora y didáctica por parte de los estudiantes.

Para la evaluación del conocimiento obtenido con la CTP, fueron comparadas las medias finales (MFs) en el módulo Histología, de los años 2013 (éstas obtenidas por medio de prueba teórica) y 2014. En el año 2014, las MFs fueron obtenidas a través de las respuestas del cuaderno de actividades (descrito en la etapa III). De acuerdo con el análisis estadístico y en comparación con las MFs en Histología del año 2013 (S) (ver tabla 2), se puede inferir que la CTP aplicada en el año 2014 fue una clase con una metodología de refuerzo excelente y diferenciado. La metodología didáctica empleada en la CTP mejoró bastante las MFS en el año 2014. Por lo tanto, la metodología pedagógica utilizada, la que (re)introdujo la acción didáctica-teórica ligada a la acción didáctica-práctica, fue capaz de mejorar la enseñanza de los sistemas auditivo y vestibular de una manera más participativa. Según los resultados (ver tabla 2), también se puede inferir que la CTP mejoró la enseñanza de los temas auditivo y vestibular, contribuyendo al conocimiento de la fisiología de los sistemas sensoriales.

Tabla 2

Análisis estadístico de las medias finales (MFs) del componente de histología de la disciplina Sistemas Sensoriales del curso de Fonoaudiología, después de la aplicación de una clase teórico-práctica diferenciada (CTP).

\begin{tabular}{lccc}
\hline Grupos analizados & $\mathrm{S}$ & $\mathrm{A} 1$ & $\mathrm{~A} 2$ \\
\hline Total de alumnos & 51 & 26 & 40 \\
Nota máxima & 8,8 & 10,0 & 10,0 \\
Nota mínima & 0,1 & 2,8 & 6,7 \\
Media & 5,6 & 6,5 & 8,6 \\
Mediana & 5,9 & 6,6 & 8,5 \\
Desviación estándar & 2,2 & 1,7 & 0,8 \\
\hline
\end{tabular}

S: alumnos del segundo semestre de 2013, sin la CTP; A1: alumnos que tuvieron la CTP en el primer semestre de 2014; A2: alumnos que tuvieron la CTP en el segundo semestre de 2014.

Para Freire (1983), el logro de la autonomía tiene un sentido socio-político-pedagógico, ya que conquistarla se vincula con la liberación de las opresiones. El logro de esta autonomía no es casual, porque es el resultado del conocimiento adquirido y el reconocimiento de luchar por ella. En este contexto, autonomía implica la libertad de pensar 
por sí mismos, lo que exige un individuo consciente y activo (Krasilchik, 2008). Basados en los comentarios de las encuestas (ej. solicitar que esta actividades fueran más frecuentes) y los resultados positivos obtenidos en la CTP creemos que la mayoría de los estudiantes adquirió el sentido de la autonomía en el aprendizaje. Lo anterior porque cada alumno se involucró con los objetos del tema que debía aprender (como el modelo 3D y preparaciones histológicas permanentes) en un intento por satisfacer su curiosidad epistemológica. Es decir, a través de su propia práctica, los estudiantes fueron entendiendo el contenido del tema tratado, a diferencia de sólo recibir la información y "guardarla en la mente". La concentración y seriedad de los estudiantes durante la CTP evidenciaron una participación autónoma de los estudiantes. En particular, sus opiniones y sugerencias mostraron que eran individuos "dueños" de sus propias actitudes. Dado lo anterior, proponemos que la CTP generó actitudes reflexivas e independientes de la relación profesor-alumno del proceso de enseñanzaaprendizaje clásico, a veces, heterónomo. Aunque la autonomía sea un atributo humano esencial, es en realidad una conquista y la educación debe proporcionar contextos formativos adecuados para que los educandos puedan hacerse autónomos.

Un objetivo importante de nuestra propuesta educacional fue promover la fijación de nuevas informaciones a través de múltiples estímulos sensoriales. En este sentido, diversos autores proponen que la actividad de conocer no está ligada a una única modalidad sensorial (visual, auditiva o táctil) aislada (Campos \& da Silva, 2001; da Silva, Morais, \& Cunha, 2011). La percepción y, aún más, la representación de cualquier objeto es un procedimiento complejo que requiere de la acción de diversos estímulos y es el resultado del funcionamiento combinado de un sistema complejo de áreas corticales, como ya hemos dicho anteriormente. Para Luria (1981), el cerebro está formado por sistemas funcionales, que se caracterizan por su complejidad estructural y por la movilidad de sus partes constituyentes. Este autor propuso sistemas funcionales integrados y no sólo de funciones. Para él, ningún proceso mental podría estar vinculado solamente a una región específica del cerebro humano o a una única modalidad sensorial. Por ello, afirma que el individuo "podría alcanzar los niveles de desarrollo y de aprendizaje por diversos caminos". En la propuesta de la CTP proporcionamos a los estudiantes un tipo de clase donde hay diversos estímulos sensoriales (estimulación visual, estimulación táctil mediante la manipulación, la estimulación mnemotécnica y la estimulación auditiva) que incorporan la propuesta de Luria (1981), en lo referente a la activación del "sistema funcional" de nuestros estudiantes para una efectiva adquisición de conocimiento.

En este contexto, el uso del modelo 3D, a diferencia de la clase teórico-práctica tradicional, buscó propiciar la activación de áreas cerebrales importantes para consolidar el aprendizaje. Estas áreas se refieren a regiones que participan en la combinación de factores tales como escuchar atentamente (estar atento a las explicaciones), observar y tocar (el modelo 3D), así como también hablar (discutir cuestiones acerca de la anatomía y la histología en el tiempo que estuvieron en contacto con el modelo). 
Escuchar atentamente es una acción que posibilita comprender lo que está siendo percibido auditivamente y procesar la información internamente (Braga, 2010). Del mismo modo, observar cuidadosamente nos permite determinar si lo que nos llamó la atención es verdaderamente relevante. De esta manera podemos desarrollar la conciencia de la escena visual y así transitar a la acción de entender, la cual nos remite a pensar sobre algo (Pinheiro, 2005). Por otro lado, el acto de tocar activa los mecanismos receptores cutáneos y subcutáneos que retransmiten la información capturada en el sistema nervioso central, que interpretará esta información mecánica y sensorial. Usando nuestros sentidos básicos conseguimos crear representaciones espacio temporales en todos los niveles del sistema somato-sensorial. Por lo tanto, mediante la exploración táctil activa (como se hace con el modelo 3D) podemos obtener una percepción detallada (discriminación táctil) de lo que tocamos (dos Santos, Del Pino, Sá-Silva, \& Pinheiro, 2017). Constantemente, examinamos objetos de forma activa con las manos para comprobar sus características, tales como la textura, la forma y el peso. Consecuentemente, nuestro cerebro comprenderá la información a través de un lenguaje no verbal muy rápido, ayudando a adquirir conocimiento sobre el objeto tocado. Por último, debatir sobre el tema, dudar sobre los planteamientos y cuestionar afirmaciones permite la reconstrucción de los conocimientos, de las capacidades cognitivas y del pensamiento, por los cuales se pueden concretar aprendizajes nuevos y elaborados (Pietrocola, 1999; Ramos \& Moraes, 2009).
La aplicación de la CTP también tuvo como objetivo la inserción entre la teoría y la actividad práctica transformadora a través de un trabajo de concientización para la acción (Demo, 2007; Ribeiro et al., 2016). Frente a las exigencias del mundo de hoy, la práctica de la enseñanza en el contexto de la sala de clases ha cambiado, para intentar ser más eficaces en la construcción del aprendizaje. Asimismo, se busca formar individuos capaces de desempeñar el papel de críticos activos de la realidad en la que operan a través del trabajo colaborativo. Esto implica un proyecto educativo interactivo e integrador entre el mediador y el estudiante, lo que ha llevado a las escuelas a buscar métodos de enseñanza participativos. Para Vygotsky (2011), el educando precisa desempeñar un papel activo en su aprendizaje, y solamente así el proceso de aprender ocurre con más rapidez y eficiencia. Para este autor, el mediador (en general, un adulto, un profesor, un especialista, un amigo o un tutor) estaría ayudando al alumno a adicionar nuevos conocimientos mediante la presentación de nuevas informaciones (Tardif, 2000). Así, en la aplicación de la CTP fue muy importante inducir un trabajo conjunto e interactivo con alumnos (aprendices), monitor (preparador estudiantil) y profesor (supervisor), no sólo para la construcción de un conocimiento específico y temático, sino también para educarlos como transformadores futuros (alumnos y monitores) de su propio aprendizaje. Además, se buscó capacitar a los estudiantes para que actuaran de manera autocrítica en su propia formación. Cabe destacar que en la CTP los monitores (preparadores estudiantiles) se encontraban en una faja etaria similar a los 
estudiantes y en la condición de "compañeros del mismo curso". Debido a esto fueron mediadores muy cercanos al alumno y su mundo. Creemos que esto posibilitó que los estudiantes se sintieran más cómodos y contribuyó a que cuestionaran los diferentes planteamientos surgidos en la CTP. Asimismo contribuyó a la asimilación de los contenidos mucho más que una clase tradicional.

\section{Conclusión}

El estudio mostró que la CTP es una apropiada actividad para el aprendizaje de la histología práctica del oído. Lo anterior, debido a que los estudiantes relacionaron de una manera efectiva la anatomía macroscópica y la estructura histológica de los tres segmentos del oído humano, lo que propició una mejor comprensión de la fisiología de los sistemas auditivo y vestibular.

Adicionalmente, la CTP fue una solución a la inadecuación de las clases anatómicas y a la inexistencia de prácticas histológicas en la disciplina $\mathrm{PCl}$ de Sistemas Sensoriales. Esta innovación didáctica se convirtió en una herramienta de gran valor y aplicabilidad, contribuyendo a un mejor aprovechamiento por parte de los alumnos del $\mathrm{PCl}$ de los Sistemas Sensoriales en la UFRJ.

Consideramos que la inclusión del modelo en 3D AS-001 en asociación con preparaciones histológicas, fue una acción innovadora que promovió estrategias metacognitivas y dinámicas donde el estudiante se transformó en un constructor de su propio aprendizaje. Es también importante destacar que la utilización de modelos anatómicos tridimensionales logró reducir el impacto emocional del uso de piezas anatómicas post-mortem.

Por lo tanto, creemos oportuna la aplicación de esta metodología innovadora de enseñanzaaprendizaje, como práctica habitual en el $\mathrm{PCl}$ de los Sistemas Sensoriales.

Nota: agradecemos al proyecto de Extensión Universitaria - Museo 3D por su valiosa contribución.

\section{Referencias}

Alarcão, I. (2015). Supervisão da Prática Pedagógica - Uma Perspectiva de Desenvolvimento e Aprendizagem - $2 a$ Edição. Leya.

Augusto, I., Monteiro, D., Girard-Dias, W., Oliveira dos Santos, T., Belmonte, S. L. R., Oliveira, J. P. de, ... Guimarães, M. C. C. (2016). Virtual Reconstruction and Three-Dimensional Printing of Blood Cells as a Tool in Cell Biology Education. PLOS ONE, 11(8), 1-13. https://doi.org/10.1371/journal.pone.0161184

Braga, C. M. D. da S. (2010). O uso de modelos no ensino da divisão celular na perspectiva da aprendizagem significativa (Mestrado Profissional). Universidade de Brasília, Brasília, DF. Recuperado de http://repositorio.unb.br/handle/10482/9069

Campos, L., \& da Silva, R. (2001). A prática como fonte de aprendizagem e o saber da experiência: o que dizem professores de Ciências e de Biologia. Investigações em Ensino de Ciências, 6(1), 79-96.

Candau, V. M. (2011). A didática em questão. Petropolis: Editora Vozes Limitada.

Correa, E. (2011). Embriologia e Histologia em Fonoaudiologia. Rio de Janeiro: Gen/Koogan.

Cunha, M. I. da. (2013). Ensino com pesquisa: a prática do professor universitário. Cadernos de Pesquisa, (97), 31-46.

da Silva, F., Morais, L., \& Cunha, I. (2011). Dificuldades dos Professores de Biologia em Ministrar aulas práticas em escolas públicas e privadas do município de Imperatriz MA. Revista Uni, 1(1), 135-149.

de Lima, G. H., da Silva, R. S., Arandas, M. J. G., de Lima Junior, N. B., Cândido, J. H. B., \& dos Santos, K. R. P. (2015). $\mathrm{O}$ uso de atividades práticas no ensino de Ciências em escolas públicas do município de Vitória de Santo Antão PE. Revista Ciência em Extensão, 12(1), 19-27. 
Demo, P. (2007). Desafios modernos da educação (14. ${ }^{\text {a }}$ ed.). Petrópolis: Vozes.

dos Santos, W. H. L., Del Pino, J. C., Sá-Silva, J. R., \& Pinheiro, R. S. (2017). A idéia do lúdico como opção metodológica no ensino de ciências e biologia: o que dizem os TCC dos egressos de ciências biológicas de licenciatura da Universidade Federal do Rio Grande do Sul? Pesquisa em foco, 21(2), 176-194. https://doi.org/10.18817/pef.v21i2.1226

Freire, P. (1983). Capítulo IV - A educação para a autonomia em Paulo Freire. Recuperado 7 de septiembre de 2018, de

http://www.pucrs.br/edipucrs/online/autonomia/autono $\mathrm{mia} /$ capitulo4.html

Krasilchik, M. (2008). Prática de Ensino de Biologia. São Paulo: EdUSP.

Luria, A. (1981). Fundamentos de Neuropsicologia. (R. Aranha, Trad.). São Paulo: Edusp.

Mor, R., \& Fragoso, M. (2012). Vestibulometria na Prática Fonoaudiológica. São José dos Campos: PULSO Editorial.

Oliveira, M. S. de, Kerbauy, M. N., Ferreira, C. N. M., Schiavão, L. J. V., Andrade, R. F. A. de, \& Spadella, M. A. (2012). Uso de material didático sobre embriologia do sistema nervoso: avaliação dos estudantes. Revista Brasileira de Educação Médica, 36(1), 83-92. https://doi.org/10.1590/S0100-55022012000100012

Pietrocola, M. (1999). Construção e Realidade: o realismo científico de Mário Bunge e o ensino de ciências através de modelos. Investigações em Ensino de Ciências. Investigações em Ensino de Ciências, 4(3), 213-227.

Pinheiro, M. (2005). Aspectos históricos da neuropsicologia: subsídios para a formação de educadores. Educar em Revista, (25), 175-196. https://doi.org/10.1590/0104-4060.372

Ramos, M., \& Moraes, R. (2009). A importância da fala na aprendizagem: Os diálogos na reconstrução do conhecimento em aulas de ciências. Presentado en VII Encontro Nacional de Pesquisa em Educaçâo em Ciências, Florianópolis-Santa Catarina, Brasil.

Ribeiro, A. S., Cardoso, A. P. G., Pereira, J. M. P., Silveira, M. G. da, Silva, S. M. da, Mariano, T. F. de S., \& Silva, L. de S. (2016). O ensino de anatomia humana no $8^{\circ}$ ano do Ensino Fundamental associado a aulas práticas mediado por ações extensionistas. En III Congresso de Ensino, Pesquisa e Extensão da UEG (CEPE) (Vol. 3, p. 9). Pirenópolis - Goiás. Recuperado de http://www.anais.ueg.br/index.php/cepe/article/view/829 $4 / 5743$
Tardif, M. (2000). Saberes profissionais dos professores e conhecimentos universitários. Revista Brasileira de Educação, 20.

Vygotsky, L. S. (2011). The Dynamics of the Schoolchild's Mental Development in Relation to Teaching and Learning. Journal of Cognitive Education and Psychology; New York, 10(2), 198-211. 